\section{A compact in vitro test bench for cardiovascular flow analysis}

Elena Torta, Giuseppe Carlo Alp Caridi, Claudio Chiastra, Diego Gallo, Umberto Morbiducci

PoliToBIOMed Lab, Department of Mechanical and Aerospace Engineering, Politecnico di Torino, Turin, Italy

\begin{abstract}
A low-cost particle image velocimetry set-up that allows to investigate the fluid dynamics inside realistic coronary artery phantoms has been implemented. The proposed smart test bench for experimental characterization of arterial hemodynamics also in the presence of implanted devices represents a low-cost equipment that can be easily implemented in non-expert laboratories for research as well as educational applications.
\end{abstract}

\section{Introduction}

Over the last two decades, Particle Image Velocimetry (PIV) applied to cardiovascular in vitro experiments have become a reliable and standard measurement technique for the evaluation of the hemodynamic performance of implantable and bloodrecirculating devices, as well as of cardiovascular surgical procedures. Advances in both hardware components and software analysis have allowed achieving major milestones in flow diagnostics, timeresolved and instantaneous volumetric measurements among the most recent ones. PIV-based experiments have been used to provide qualitative and quantitative visualizations both of laminar and turbulent fluid structures characterizing biological flow. A limiting factor in using PIV-based systems is represented by their cost: PIV components, i.e. high-power laser, research-level cameras and synchronisation units, can be extremely expensive for most academic and industrial laboratories (order of $100 \mathrm{k} €$ in total). In addition, pulsed lasers commonly used in PIV pose safety concerns and require strict safety procedures and standards (EN 207 in EU; ANSI z136 in US). In recent years, attempts have been made to use smart-PIV systems to overcome the limitations mentioned above. 1,2 In this respect, the imaging equipment of commercial smartphones, coupled with low-energy light sources, has recently proved to be a suitable component for PIV measurements in air and water flows. ${ }^{1}$

In vitro modelling with artificial flow phantoms (scale 1:1) has been used to investigate the fluid mechanics of the circulatory system without the ethical and safety issues associated with animal and human experiments. ${ }^{3}$ Here we propose a smart test bench based on the use of a smartphone camera and low-power laser for flow characterization in realistic phantoms of coronary arteries. The compactness of the proposed test bench makes its suitable for industrial applications as well as for those research applications where miniaturization is necessary, such as in space missions. ${ }^{3}$

\section{Materials and Methods}

Preliminary tests were performed to demonstrate the feasibility of a compact, inexpensive optical imaging system applied to the study of coronary flows (Figure 1). The experiments were carried out on two realistic 3D-printed silicone coronary phantoms obtained from angiographic images, the first representing a stenotic left circumflex (LCX) coronary artery, the second reproducing the same artery in the healthy condition.

Measurements were conducted monitoring flow rates (in the range 30-90 $\mathrm{mL} / \mathrm{min}$ ) using an ultrasonic flow meter (Transonic, Ithaca, USA) and pressure losses $(\Delta \mathrm{p})$ through two pressure sensors (BDC Laboratories, Wheat Ridge, USA). A Samsung galaxy S9+ (pixel size $1.4 \mathrm{~mm}$ ) camera was embedded in the system for time-resolved image acquisition (190x730 pixel at $960 \mathrm{~Hz}$ ) of the fluid domain. The coronary phantoms were illuminated using a continuous laser $(30 \mathrm{~mW}, 520 \mathrm{~nm})$. A
Correspondence: Elena Torta, PoliTo ${ }^{\mathrm{BIO}} \mathrm{Med}$ Lab, Department of Mechanical and Aerospace Engineering, Politecnico di Torino, Turin, Italy.

E-mail: elena.torta@polito.it

Key words: Atherosclerosis; coronary artery; low-cost particle image velocimetry; smartphone.

Disclosures: The authors have no professional or financial conflicts of interest to disclose.

Conference presentation: This paper was presented at the Third Centro 3R Annual Meeting - L'era delle 3R: modelli in silico, in vitro e in vivo per promuovere la ricerca traslazionale 30 September - 1 October 2021, Evento online organizzato dal Politecnico di Torino.

Received for publication: 9 July 2021.

Accepted for publication: 7 September 2021.

This work is licensed under a Creative Commons Attribution NonCommercial 4.0 License (CC BY-NC 4.0).

(c) Copyright: the Author(s), 2021

Licensee PAGEPress, Italy

Biomedical Science and Engineering 2021; 4(s1):196 doi:10.4081/bse.2021.196

blood-mimicking fluid (glycerol-water, 33:67) was seeded with polyamide particles $\left(\mathrm{r}=1.1 \mathrm{~g} / \mathrm{cm}^{3}, d_{p}=2 \mu \mathrm{m}\right)$. The velocity field, obtained with the open-source code PIVlab, 5 had a spatial resolution of 0.035 $\mathrm{mm}$. The experimental set-up was validated comparing the measured velocity profiles with Poiseuille law in a cylindrical pipe with the same dimension of the healthy coronary phantom $(\mathrm{D}=3 \mathrm{~mm})$ at Reynolds ranging from 76 to 230 .

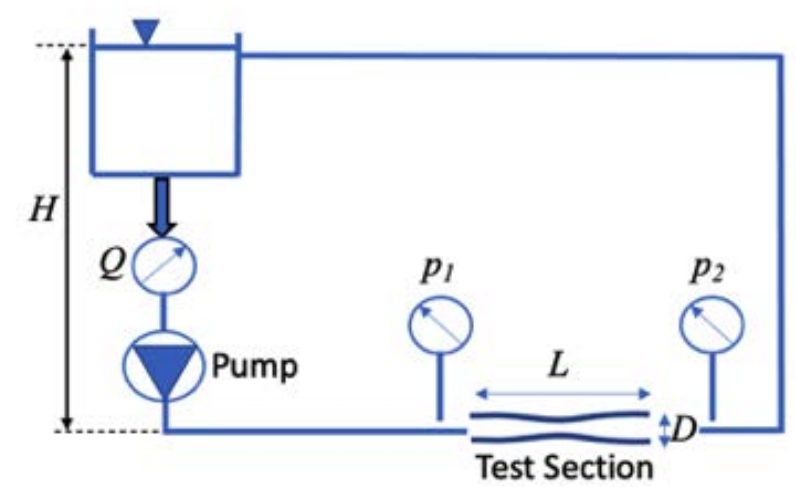

Figure 1. Experimental set up. 
confirm that the spatial resolution of the system is sufficient to capture the main flow features characterizing the coronary hemodynamics, at different flow conditions. In detail, flow measurements highlight the expected presence of high spatial velocity gradients in the stenosis region and of a post-stenotic recirculation region whose extension is dictated by the investigated flow regime (data not shown). wall uncertainty is a limitation affecting also conventional PIV measurements, a consequence of light reflections and limited spatial resolution.

The velocity vector fields in the healthy and stenotic vessels presented in Figure 2b,

\section{Discussion and Conclusions}

The findings of the study demonstrate (a)

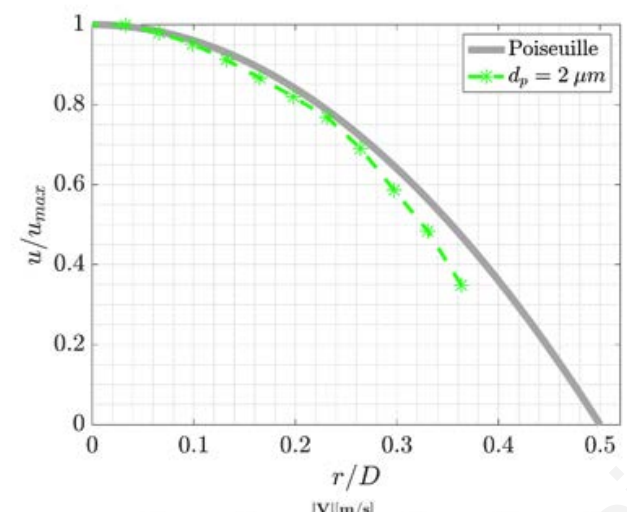

(b)
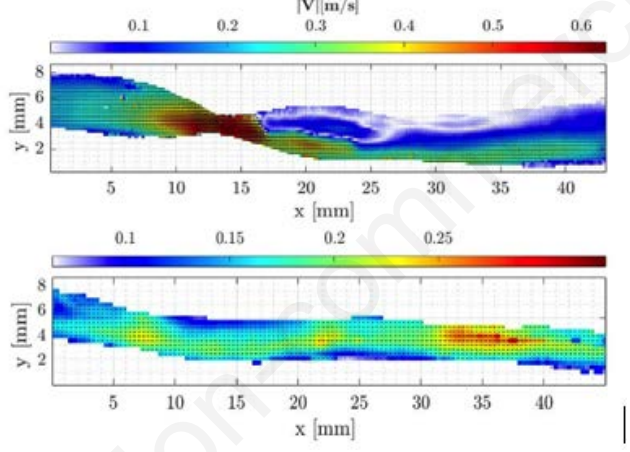

Figure 2. (a) Comparison between experimental and analytical velocity profiles $(Q=30$ $\mathrm{ml} / \mathrm{min}$ ). (b) Average velocity vector field in the stenotic (top) and healthy (bottom) phantom. The fluid flows from the left to the right $(Q=70 \mathrm{ml} / \mathrm{min})$. that the proposed low-cost PIV system provides velocity fields with resolution sufficient to capture the main coronary flow features. The system presented in this study candidates to be a compact, reliable, lowcost instrument for qualitative and quantitative flow diagnostics in the biomedical field. It could potentially be employed to perform PIV analyses on patient-specific cardiovascular 3D-printed phantoms testing, e.g. surgical strategies before interventions, or for the evaluation of the hemodynamic performances of implantable cardiovascular devices, e.g. stent. The compactness of the proposed set up candidates the proposed approach to future applications where miniaturized test-bench are mandatory, making it possible for the first time cardiovascular flow characterization e.g. in experiments designed for space missions such as the $2 \mathrm{U}$ experiment cube Artery in Microgravity project. ${ }^{4}$

\section{References}

1. Cierpka C, Hain R, Buchmann NA. Flow visualization by mobile phone cameras. Exp Fluids 2016;57:108.

2. Käufer T, König J, Cierpka C. Stereoscopic PIV measurements using low-cost action cameras. Exp Fluids 2021;62:57.

3. Geoghegan PH, Buchmann NA, Soria J, et al. Time-resolved PIV measurements of the flow field in a stenosed, compliant arterial model. Exp Fluids 2013;54:1528.

4. Drayson O, Berbnardini N, Abderrahaman AB, et al. Aim (artery in microgravity): Design and development of an ice cubes experiment. 70th International Astronautical Congress, IAC 2019, Washington, 21 October 2019 - 25 October 2019. Article number IAC-19 A2 $6 \quad 8 \quad$ x50731

5. Thielicke $\mathrm{W}, \overline{\text { Sonntag R.Particle Image }}$ Velocimetry for MATLAB: Accuracy and enhanced algorithms in PIVlab. J Open Res Software 2021;9:12. 